\title{
Series Solution of Three Dimensional Peristaltic Flow of Prandtl Fluid in a Rectangular Channel
}

S Nadeem ${ }^{1 *}$, Arshad Riaz ${ }^{2}$ and R Ellahi ${ }^{2}$

${ }^{1}$ Department of Mathematics, Quaid-i-Azam University 45320, Islamabad 44000, Pakistan

${ }^{2}$ Department of Mathematics and Statistics, FBAS, IIU, Islamabad, 44000, Pakistan

\begin{abstract}
The study of peristaltic flow of an incompressible non-Newtonian Prandtl fluid model has been explained in three dimensional rectangular channels. All the corresponding equations have been derived in three dimensional coordinate system. The governing equations have been solved analytically to achieve the expressions for velocity and pressure gradient while pressure rise data has been acquired numerically. The physical aspects of appertaining quantities have been enlightened through graphical assistance in both two and three dimensions. The stream functions have also been delineated for all the inclusive parameters.
\end{abstract}

Keywords: Peristaltic flow; Prandtl fluid; Rectangular channel; Analytical and numerical solutions

\section{Introduction}

Peristaltic flow of non-Newtonian fluids have attracted the exclusive meditation of majority of the mathematicians, physicists and chemical engineers due to the wide range of applications and implementations of such type of flows in industry, technology, physiology and biological fields. A large class of real fluids does not exhibit the linear relationship between stress and the rate of strain. Because of the non-linear dependence, the analysis of the behavior of the fluid motion of the non-Newtonian fluids tends to be much more complicated and subtle in comparison with that of the Newtonian fluids. In the literature, the mechanics of non-linear fluids presents special challenges to engineers, physicists and mathematicians since the non-linearity can manifest itself in a variety of ways [1]. A vast amount of literature is available on the topic. Some of them can be cited in refs. [2-5]. The studies of peristaltic flows of Newtonian and non-Newtonian fluid have been investigated by many researchers [6-10].

The above discussed studies relating peristaltic flows are investigated in two dimensional geometries. Nowadays, researcher have decided to work on the ...led of peristalsis in three dimensional channels and tubes. Reddy et al. [11] have analyzed the influence of lateral walls on peristaltic flow in a rectangular duct and laid down the idea that the sagittal cross section of the uterus may be better approximated by a three dimensional channel of rectangular cross section as compared with a two dimensional channel. Mekheimer et al. [12] has recently published the mathematical model of peristaltic transport through eccentric cylinders. Stokesian peristaltic pumping in a three-dimensional tube with a phase-shifted asymmetry has been undertaken by Vivian et al., [13]: Tang [14] have considered the three dimensional numerical and asymptotic solutions for the peristaltic transport of a heat-conducting fluid and have observed that the relation between mass flux and pressure drop remains almost linear and the efficiency of the transport depends mainly on the ratio of the wave amplitude and the average radius of the tube. The idea of peristaltic flow of non-Newtonian Prandtl fluid has not been revealed in a three dimensional channel by any researcher till now.

The intent of the present investigation is to measure the peristaltic flow of Prandtl fluid in a three dimensional rectangular cross section. The equations for the conservation of mass and momentum have been obtained in three dimensional Cartesian coordinate system. The approximations of low Reynolds number and long wavelength have been incorporated to reduce the complexity of the observing problem. The flow is considered in a wave frame of reference transferring with a speed $c$ along the channel walls. The expressions for velocity and pressure gradient have been solved analytically and pressure rise relation is evaluated numerically. The data obtained for velocity profile, pressure gradient and pressure rise has been portrayed graphically to inquire the influence of all pertinent parameters accruing in the problem. Trapping scheme is also presented to encompass the physical features of observing parameters on the stream functions.

\section{Mathematical Formulation}

Let us analyze the peristaltic flow of incompressible Prandtl fluid in a cross section of rectangular channel enclosing the width $2 \mathrm{~d}$ and height 2a. To proceed the analysis, Cartesian coordinate system is incorporated in such a way that $\mathrm{x}$ axis is taken along the axial direction, $y$ axis is taken along the lateral direction and $\mathrm{z}$ axis is along the normal way of rectangular channel (Figure 1). The flow is produced by the peristaltic sinusoidal waves propagating along the direction of the flow.

The boundaries of the peristaltic walls of the channel are presented as

$$
Z=H(X, t)= \pm a \pm b \cos \left[\frac{2 \Pi}{\lambda}(X-c t)\right]
$$

Where $\mathrm{a}$ and $\mathrm{b}$ are the amplitudes of the waves, $\lambda$ is the wavelength, $\mathrm{c}$ is the velocity of propagation, $\mathrm{t}$ is the time and $\mathrm{x}$ is the direction of wave propagation. The walls parallel to xz-plane remain stable and do not cause any peristaltic wave motion. The stress tensor for the Prandtl fluid model is defined by Patel and Timol [15]

*Corresponding author: S Nadeem, Department of Mathematics, Quaid-i-Azam University 45320, Islamabad 44000, Pakistan, Tel: 92 3339822640; E-mail: ariiui@hotmail.com

Received December 26, 2013; Accepted February 13, 2014; Published February 25,2014

Citation: Nadeem S, Riaz A, Ellahi R (2014) Series Solution of Three Dimensional Peristaltic Flow of Prandtl Fluid in a Rectangular Channel. J Appl Mech Eng 3: 139. doi:10.4172/2168-9873.1000139

Copyright: (c) 2014 Nadeem S, et al. This is an open-access article distributed under the terms of the Creative Commons Attribution License, which permits unrestricted use, distribution, and reproduction in any medium, provided the original author and source are credited. 


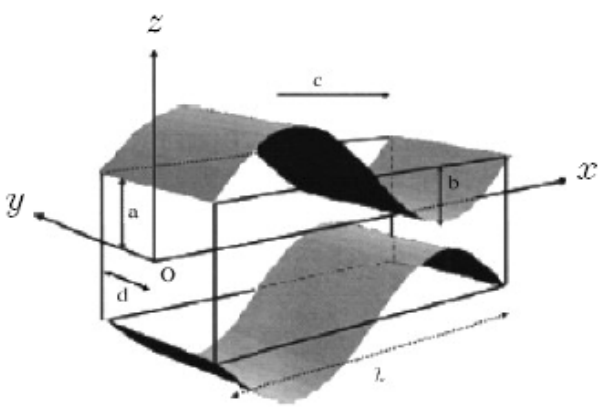

Figure 1: Schematic diagram for peristaltic flow in a rectangular duct.

$$
\tau=\frac{A \sin ^{-1}\left(\frac{1}{c}\left(\left(\frac{\partial U}{\partial Y}\right)^{2}+\left(\frac{\partial W}{\partial Y}\right)^{2}\right)^{1 / 2}\right)}{\left(\left(\frac{\partial U}{\partial y}\right)^{2}+\left(\frac{\partial W}{\partial y}\right)^{2}\right)^{1 / 2}} \frac{\partial U}{\partial Y},
$$

in which A and C represent material constants of Prandtl fluid model. The velocity field for the present flow problem will be $(\mathrm{U} ; 0 ; \mathrm{W})$ in a rectangular duct. The governing equations for the flow problem are stated as

\section{Conservation of mass}

$$
\frac{\partial U}{\partial X}+\frac{\partial W}{\partial Z}=0
$$

\section{Conservation of momentum}

$$
\begin{aligned}
& \rho\left(\frac{\partial U}{\partial t}+U \frac{\partial U}{\partial X}+W \frac{\partial U}{\partial Z}\right)=-\frac{\partial P}{\partial X}+\frac{\partial}{\partial X} \tau_{X X}+\frac{\partial}{\partial X} \tau_{X Y}+\frac{\partial}{\partial X} \tau_{X Z}, \\
& 0=-\frac{\partial P}{\partial Y}+\frac{\partial}{\partial X} \tau_{Y X}+\frac{\partial}{\partial X} \tau_{Y Y}+\frac{\partial}{\partial X} \tau_{Y Z}, \\
& \rho\left(\frac{\partial W}{\partial t}+U \frac{\partial W}{\partial X}+W \frac{\partial W}{\partial Z}\right)=-\frac{\partial P}{\partial Z}+\frac{\partial}{\partial X} \tau_{Z X}+\frac{\partial}{\partial Y} \tau_{Z Y}+\frac{\partial}{\partial Z} \tau_{Z Z},
\end{aligned}
$$

in above relations, $\rho$ is the density, $\mathrm{P}$ is the pressure. Let us define a wave frame $(x, y)$ moving with the velocity $c$ away from the fixed frame $(\mathrm{X}, \mathrm{Y})$ by the following transformation

$$
x=X-c t, y=Y, z=Z, u=U-c, w=W, p(x, y, z)=P(X, Y, Z, t)
$$

Defining the following non-dimensional quantities

$$
\begin{aligned}
& \bar{x}=\frac{x}{\lambda}, \bar{y}=\frac{y}{d}, \bar{z}=\frac{z}{a}, \bar{u}=\frac{u}{c}, \bar{w}=\frac{w}{c \delta}, \bar{t}=\frac{c t}{\lambda}, \bar{h}=\frac{H}{a}, \bar{p}=\frac{a^{2} p}{\mu c \lambda}, \\
& \operatorname{Re}=\frac{\rho a c}{\mu}, \delta=\frac{a}{\lambda}, \phi=\frac{b}{a}, \bar{\tau}=\frac{a}{\mu c} \tau, \beta=\frac{a}{d}, \alpha=\frac{A}{\mu c}, \beta_{1}=\frac{\alpha c^{2}}{6 C^{2} a^{2}},
\end{aligned}
$$

Where $\phi$, Re and $\delta$ describe the amplitude ratio, the Reynold's number and the dimensionless wave number,respectively. The quantities $\alpha$ and $\beta_{1}$ demonstrate the dimensionless parameters of Prandtl fluid. Utilizing the above non-dimensional quantities into equations (2) to (6), the resulting equations (after dropping the bars) in wave frame can be written as

$$
\frac{\partial u}{\partial x}+\frac{\partial w}{\partial z}=0
$$

$$
\begin{aligned}
& \operatorname{Re} \delta\left(u \frac{\partial u}{\partial x}+w \frac{\partial u}{\partial z}\right)=-\frac{\partial p}{\partial x}+\delta \frac{\partial}{\partial x} \tau_{x x}+\beta^{2} \frac{\partial}{\partial y} \tau_{x y}+\frac{\partial}{\partial z} \tau_{x z}, \\
& 0=-\frac{\partial p}{\partial y}+\delta^{2} \frac{\partial}{\partial x} \tau_{y x}+\delta^{2} \frac{\partial}{\partial y} \tau_{y y}+\delta \frac{\partial}{\partial z} \tau_{y z} \\
& \operatorname{Re} \delta^{2}\left(u \frac{\partial w}{\partial x}+w \frac{\partial w}{\partial z}\right)=-\frac{\partial p}{\partial z}+\delta^{2} \frac{\partial}{\partial x} \tau_{z x}+\delta \beta^{2} \frac{\partial}{\partial y} \tau_{z y}+\delta^{2} \frac{\partial}{\partial z} \tau_{z z},
\end{aligned}
$$

Applying the approximations of long wave length $(\lambda \rightarrow \infty)$ and low Reynolds number $(\operatorname{Re} \rightarrow 0$ ), the governing equation for the Prandtl fluid in non-dimensional form is described as

$$
\frac{1}{\alpha} \frac{\partial p}{\partial x}=\beta^{2} \frac{\partial^{2} u}{\partial y^{2}}+\frac{\partial^{2} u}{\partial z^{2}}+\frac{3 \beta_{1}}{\alpha}\left(\beta^{4}\left(\frac{\partial u}{\partial y}\right)^{2} \frac{\partial^{2} u}{\partial y^{2}}+\left(\frac{\partial u}{\partial z}\right)^{2} \frac{\partial^{2} u}{\partial z^{2}}\right)
$$

The corresponding non-dimensional boundary conditions for the peristaltic flow in rectangular channel are stated as

$$
\begin{aligned}
& u=-1 \text { at } y= \pm 1 \\
& u=-1 \text { at } z= \pm h(x, t)= \pm 1 \pm \phi \cos 2 \prod x
\end{aligned}
$$

It is to be noted here if $\beta \rightarrow 0$ the present problem reduces to Prandtl model for two dimensional channels and $\beta=1$ gives the square duct.

\section{Solution of the Problem}

The solution of the nonlinear partial differential equation (12) along with boundary conditions (13) and (14) have been calculated by analytical series solution technique which is defined as $[16,17]$

$$
u_{1}=\sum_{n=1}^{\infty} a_{n}(y) \cos (2 n-1) \frac{\pi}{2 h} z,
$$

in which $\mathrm{q}$ is the embedding parameter having the range $0 \leq q \leq 1$.

We have chosen $L=\beta^{2} \frac{\partial^{2}}{\partial y^{2}}+\frac{\partial^{2}}{\partial z^{2}}$ as the linear operator. We choose the following initial guess

$$
\widehat{u}_{0}(y, z)=-1+\frac{1}{\beta^{2}}\left(1-y^{2}\right)+z^{2}-h^{2}
$$

Applying perturbation on the embedding parameter $\mathrm{q}$ for the variable $v$, we have

$$
v(x, y, z, q)=u_{0}+q u_{1}+q^{2} u_{2}+\ldots .
$$

Substituting equation (17) into equation (15) and then comparing the like powers of $\mathrm{q}$ one obtains the following problems together with the corresponding boundary conditions.

\section{Zeroth order deformation}

$$
\begin{aligned}
& L\left(u_{0}\right)-L\left(\widehat{u_{0}}\right)=0, \\
& u_{0}=-1 \text { at } y= \pm 1, \\
& u_{0}=-1 \text { at } z= \pm h(x) .
\end{aligned}
$$

From equations (16) and (18) we have the following zeroth order solution

$$
u_{0}=\widehat{u_{0}}(y, z)=-1+\frac{1}{\beta^{2}}\left(1-y^{2}\right)+z^{2}-h^{2} .
$$


Citation: Nadeem S, Riaz A, Ellahi R (2014) Series Solution of Three Dimensional Peristaltic Flow of Prandtl Fluid in a Rectangular Channel. J Appl Mech Eng 3: 139. doi:10.4172/2168-9873.1000139

Page 3 of 7

\section{First order deformation}

$$
\beta^{2} \frac{\partial^{2} u_{1}}{\partial y^{2}}+\frac{\partial^{2} u_{1}}{\partial z^{2}}+\frac{3 \beta_{1}}{\alpha}\left(\beta^{4}\left(\frac{\partial u_{0}}{\partial y}\right)^{2} \frac{\partial^{2} u_{0}}{\partial y^{2}}+\left(\frac{\partial u_{0}}{\partial z}\right)^{2} \frac{\partial^{2} u_{0}}{\partial z^{2}}\right)-\frac{1}{\alpha} \frac{\partial p}{\partial x}=0
$$

Using equation (21) into above equation, we have the following relation

$$
\beta^{2} \frac{\partial^{2} u_{1}}{\partial y^{2}}+\frac{\partial^{2} u_{1}}{\partial z^{2}}=\frac{24 \beta_{1}}{\beta^{2} \alpha}\left(y^{2}-\beta^{2} z^{2}\right)+\frac{1}{\alpha} \frac{\partial p}{\partial x},
$$

Now incorporating the method of eigen function expansion, we obtain the following series solution

$$
u_{1}=\sum_{n=1}^{\infty} a_{n}(y) \cos (2 n-1) \frac{\pi}{2 h} z
$$

Where an $(y)$ is evaluated as $a_{n}(y)=-\left(4 \exp \left[\lambda_{n} / \beta\right] h(x)(-1)^{n}\left(\left(-192 h^{2}(x) \beta^{2} \beta_{1} \lambda_{n}^{2}+\pi^{2}(1-2 n)^{2}\left(-48 \beta^{2} \beta_{1}\right)\right.\right.\right.$

$\left.\left.-\left(d p / d x \beta^{2}+24\left(y^{2}-h^{2}(x) \beta^{2}\right) \beta_{1}\right) \lambda_{n}^{2}\right)\right) \cosh \left(\lambda_{n} / \beta\right)+\left(192 h^{2}(x) \beta^{2}\right.$

$\left.\beta_{1} \lambda_{n}^{2}-\pi^{2}(1-2 n)^{2}\left(-48 \beta^{2} \beta_{1}-\left(24 \beta_{1}+\beta^{2}\left(d p / d x-24 h^{2}(x) \beta_{1}\right)\right) \lambda_{n}^{2}\right)\right)$

$\left.\left.\cosh \left(\lambda_{n} y / \beta\right)\right)\left(1-\tanh \left(\lambda_{n} / \beta\right)\right)\right) /\left((2 n-1)^{3} \pi^{3} \alpha \beta^{2} \lambda_{n}^{4}\right.$

Here, $\lambda_{n}=(2 n-1) \pi / 2 h, n=1,2,3, \ldots \ldots$ are the eigen values.

According to the solution scheme, the nal solution is acquired at $\mathrm{q} \rightarrow 1$ and is de.ned as

$$
\mathrm{u}(\mathrm{x}, \mathrm{y}, \mathrm{z})=\mathrm{u} 0+\mathrm{u} 1+\ldots \ldots,
$$

where $\mathrm{u}_{0}$ and $\mathrm{u}_{1}$ are displayed in Equations (21) and (24). The volumetric flow rate $\mathrm{F}$ is represented by the following relation



(a)

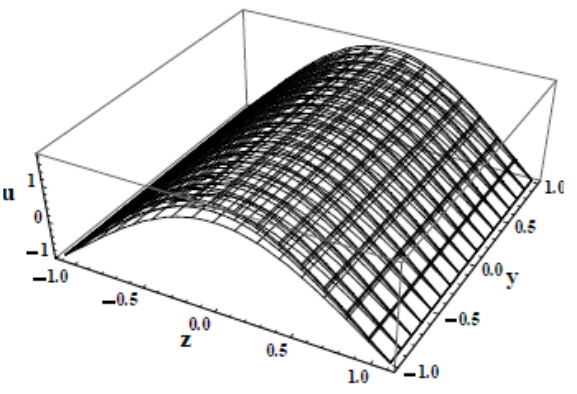

(b)

Figure 2: Velocity profile for different values of $\beta$ for fixed $\phi=0.1, x=0, \alpha=0.2, \beta_{1}=0.1, Q=1,(a)$ for 2 dimensional, (b) For 3 dimensional.

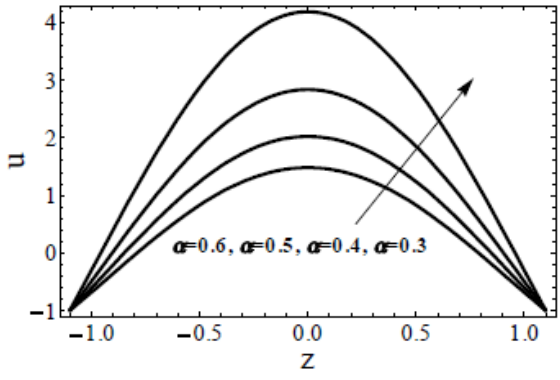

(a)

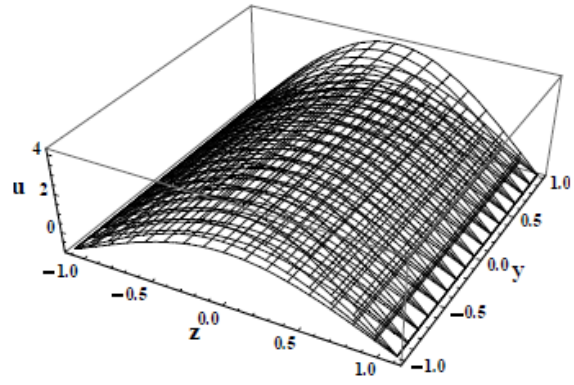

(b)

Figure 3: Velocity profile for different values of $\alpha$ for fixed $\phi=0: 1, x=0, \beta=0.7, \beta_{1}=0.9, Q=1$, (a) For 2 dimensional, (b) For 3 dimensional.



(a)

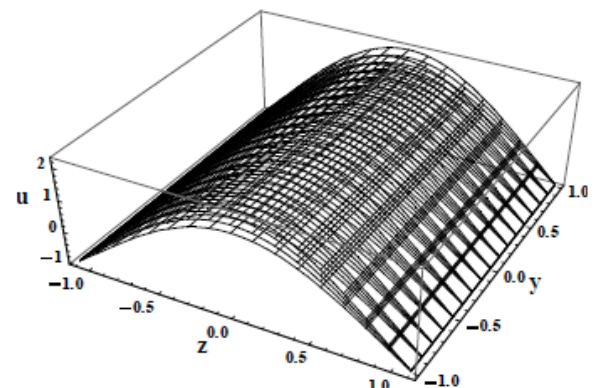

(b)

Figure 4: Velocity profile for different values of $\beta_{1}$ for fixed $\phi=0.1, x=0, \alpha=0.2, \beta=1.5, Q=1$, (a) For 2 dimensional, (b) For 3 dimensional. 


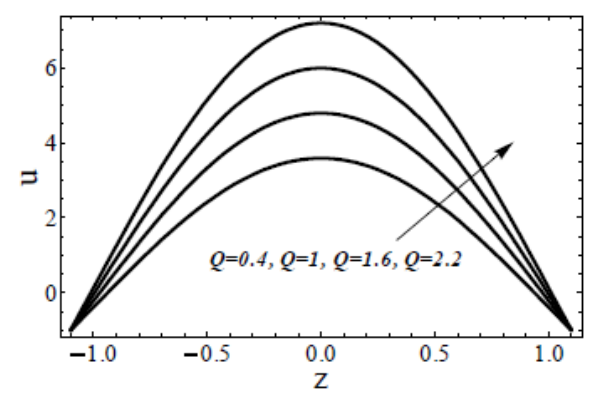

(a)



(b)

Figure 5: Velocity profile for different values of $Q$ for fixed $\phi=0.1, x=0, \alpha=0.6, \beta=2, \beta=0.7$, (a) For 2 dimensional, (b) For 3 dimensional.

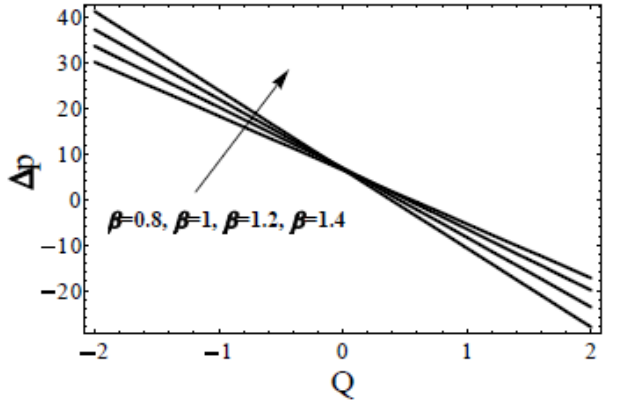

Figure 6: Variation of pressure rise $\Delta p$ for different values of $\beta$ for fixed $\phi=0.5, \alpha=0.7, \beta_{1}=0.9, y=0: 1$.

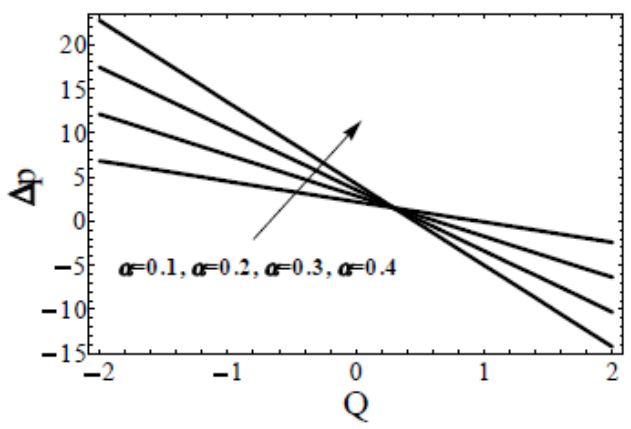

Figure 7: Variation of pressure rise $\Delta p$ for different values of $\alpha$ for fixed $\phi=0.5, \beta=1.3, \beta_{1}=0.7, y=0.1$.

$F=\int_{0}^{h(x)} \int_{0}^{1} u(x, y, z) d y d z$

The instantaneous flux is given by

$\bar{Q}=\int_{0}^{h(x)} \int_{0}^{1} u+1 d y d z=F+h(x)$

The average volume flow rate over one period $(\mathrm{T}=\lambda / \mathrm{c})$ of the peristaltic wave is defined as

$$
Q=\frac{1}{T} \int_{0}^{T} \bar{Q} d t=F+h(x)
$$

The pressure gradient $\mathrm{dp} / \mathrm{dx}$ is executed after solving equations (27) and (29) and is written as

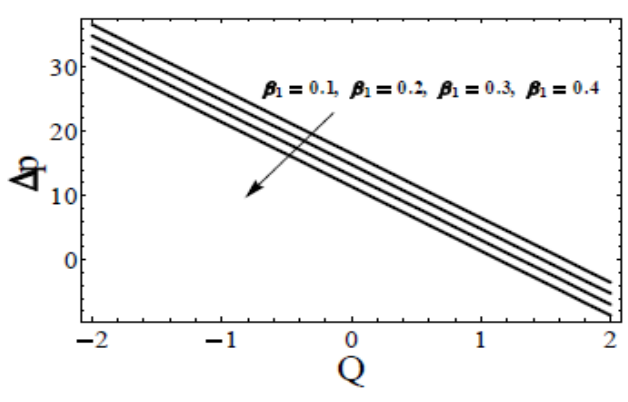

Figure 8: Variation of pressure rise $\Delta p$ for different values of $\beta_{1}$ for fixed $\phi=0.5, \beta=0.5, \alpha=0.7, y=0.1$.

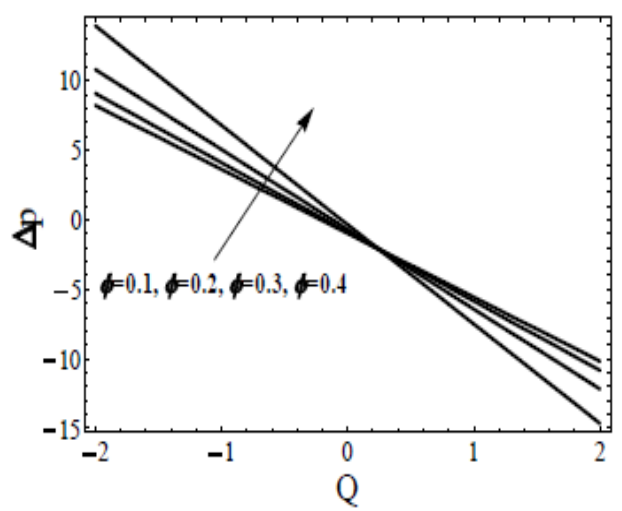

Figure 9: Variation of pressure rise $\Delta p$ for different values of $\phi$ for fixed $\alpha=0.3, \beta=1.5, \beta_{1}=0.9, y=0.1$

$$
\begin{aligned}
& \frac{d p}{d x}=\left(( - 1 ) ^ { 2 n } \left(\lambda _ { n } \left(576 h^{2}(x)(1-2 n)^{2} \pi^{2} \beta^{2} \beta_{1}-96 h^{2}(x)\left(-24 h^{2}(x) \beta^{2}+(1-2 n)^{2}\right.\right.\right.\right. \\
& \left.\pi^{2}\left(3 h^{2}(x) \beta^{2}-1\right)\right) \beta_{1} \lambda_{n}^{2}+(1-2 n)^{4} \pi^{4} \alpha\left(-2 h(x)+\left(3 h(x)-3+2 h^{3}(x)+3 Q\right) \beta^{2}\right. \\
& \lambda_{n}^{4}-96 h^{2}(x) \beta_{1}\left(-24 h^{2}(x) \beta^{2} \lambda_{n}^{2}+\pi^{2}(1-2 n)^{2}\left(-6 \beta^{2}+\left(3 h^{2}(x) \beta^{2}-1\right) \lambda_{n}^{2}\right)\right)(-1)^{2 n} \\
& \cosh \left(\frac{\lambda_{n}}{\beta}\right)+576 h^{2}(x) \beta \beta_{1}\left(-8 h^{2}(x) \beta^{2} \lambda_{n}^{2}+\pi^{2}(1-2 n)^{2}\left(-2 \beta^{2}+\left(h^{2}(x) \beta^{2}-1\right) \lambda_{n}^{2}\right)\right) \\
& \left.\left.\sinh \left(\frac{\lambda_{n}}{\beta}\right)\right)\right) /\left(24 h^{2}(x) \pi^{2}(1-2 n)^{2} \beta^{2} \lambda_{n}^{2}\left(-\lambda_{n} \cosh \left(\frac{\lambda_{n}}{\beta}\right)+\beta \sinh \left(\frac{\lambda_{n}}{\beta}\right)\right)\right)
\end{aligned}
$$

The pressure rise $\Delta \mathrm{p}$ is evaluated numerically by using the following integral

$$
\Delta p=\int_{0}^{1} \frac{d p}{d x} d x
$$




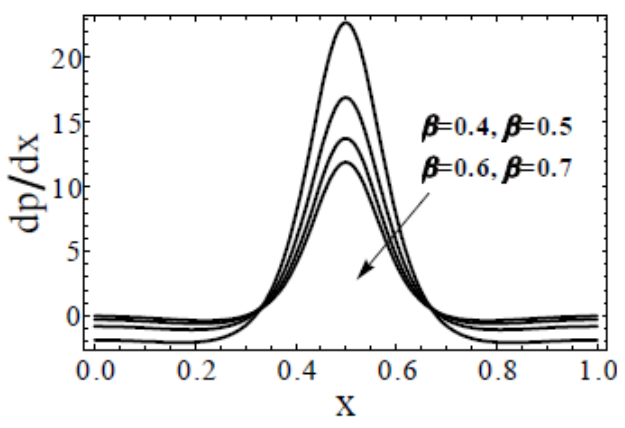

Figure 10: Pressure gradient distribution $\mathrm{dp} / \mathrm{dx}$ for different values of $\beta$ for fixed $\alpha=0.1, \phi=0.6, \beta_{1}=0.1, y=1 ; Q=0.2$.

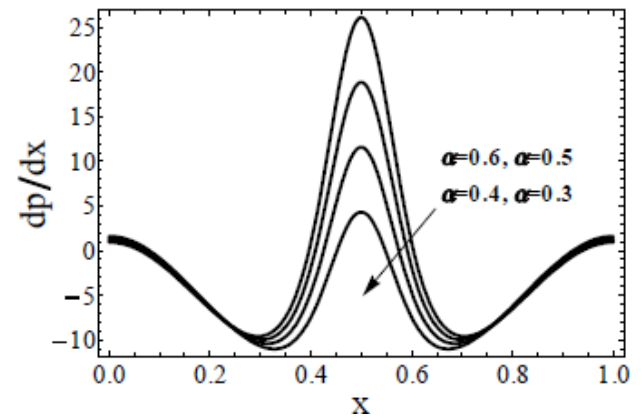

Figure 11: Pressure gradient distribution $\mathrm{dp} / \mathrm{dx}$ for different values of $\alpha$ for fixed $\beta=0.7, \phi=0.6, \beta_{1}=0.5, y=1 ; Q=0.6$.

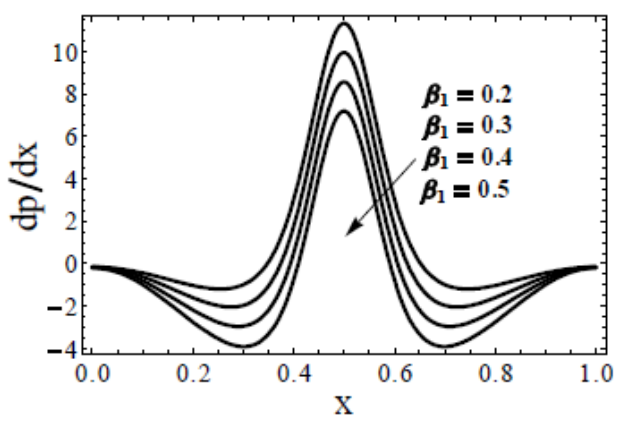

Figure 12: Pressure gradient distribution $\mathrm{dp} / \mathrm{dx}$ for different values of $\beta 1$ for fixed $\beta=0.65, \phi=0.6, \alpha=0.1, y=1 ; Q=0.2$.

\section{Graphical Results and Discussions}

Graphical aspects of pertinent parameters for the distributions of velocity, pressure rise, pressure gradient and stream function are portrayed in the present discussion. The highly non-linear partial differential equations have been solved analytically. Three dimensional features of the velocity variation are also pointed out. Figures 2-5 are plotted to investigate the influence of aspect ratio $(\beta)$, Prandtl fluid parameters $(\alpha, \beta 1)$ and average volume flow rate $Q$ on the velocity profile $\mathrm{u}$. Pressure rise $\Delta \mathrm{p}$ is sketched against the flow rate $\mathrm{Q}$ for thequantities aspect ratio $(\beta)$, Prandtl fluid parameters $\left(\alpha, \beta_{1}\right)$ and amplitude ratio $\phi$ in Figures 6-9. One can extract the behavior of pressure gradient curves $\mathrm{dp} / \mathrm{dx}$ under the variation of $\beta, \alpha, \beta_{1}$ and $\mathrm{Q}$ from Figures 10-13. The process of trapping bolus is also manipulated through presenting streamlines for above discussed parameters.

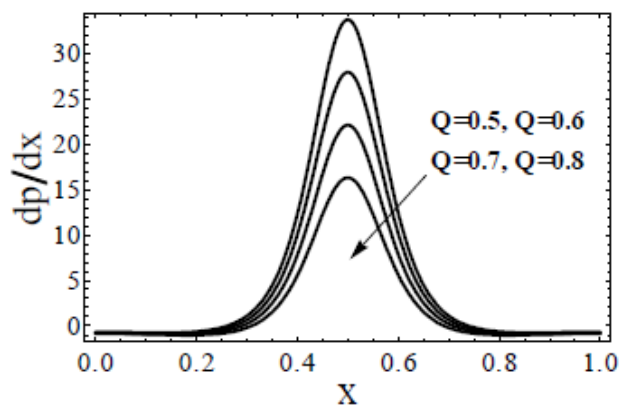

Figure 13: Pressure gradient distribution $\mathrm{dp} / \mathrm{dx}$ for different values of $Q$ for fixed $\beta=0.7, \phi=0.6, \beta_{1}=0.1, y=1 ; Q=0.4$.

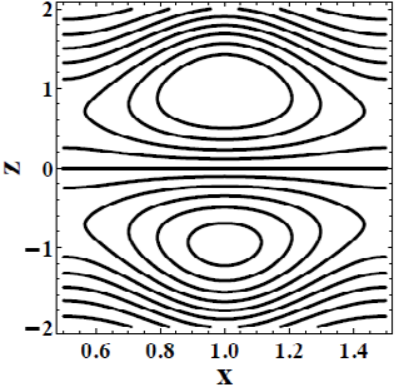

(a)

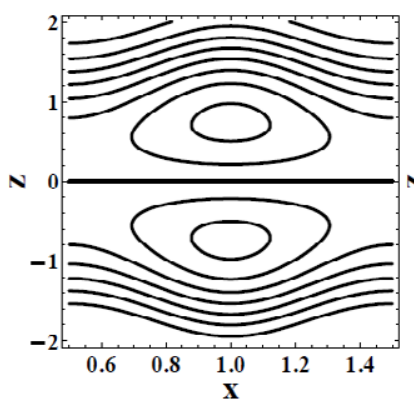

(c)

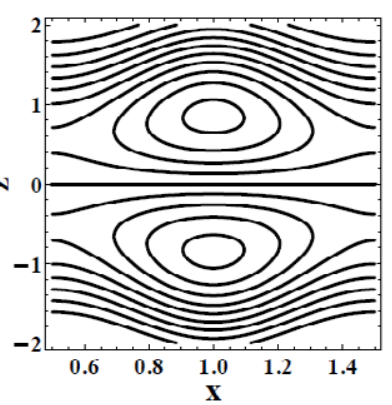

(b)

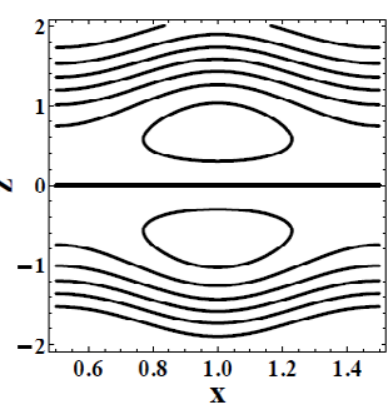

(d)
Figure 14: Stream lines for different values of $\beta(a)$ for $\beta=0.5$, (b) for $\beta=0.6$, (c) for $\beta=0.7$, (d) for $\beta=0.8$. The other parameters are $y=0.3, \alpha=0.2, \beta_{1}=0.5$, $\mathrm{Q}=1, \phi=0.1$.

Figure $2 \mathrm{a}, 2 \mathrm{~b}$ includes the variations of velocity profile $\mathrm{u}$ against the axial direction $\mathrm{z}$ for the aspect ratio $\beta$ when $\phi=0.1, \alpha=0.2, \beta_{1}=0.1, \mathrm{Q}=1$. One can imagine from the figure that velocity profile is rising when aspect ratio is increased to large values and approaches its highest peak at the centre of the flow. We can observe from Figure 3 that velocity is drawn to visualize the impact of Prandtl fluid parameter $\alpha$ when other parameters are kept fixed i.e. $\phi=0.1, \beta=0.7, \beta_{1}=0.9, Q=1$. It can be seen here that profile of the velocity is descending when someone enlarges the magnitude of $a$ which shows that flow is slowed down with the more quantity of $\alpha$. From Figures 4 and 5 we can conclude that velocity distribution is directly varying with the variation of parameter $\beta_{1}$ and the flow rate $\mathrm{Q}$, respectively. It also discloses that velocity gets maximum height at the point $\mathrm{z}=0$ and velocity increases in the region $-1<\mathrm{z}<0$ but decreasing in the remaining part i.e. $0<\mathrm{z}<1$ with both the parameters.

The pressure rise $\Delta \mathrm{p}$ for the aspect ratio $\beta$ is portrayed in Figure 


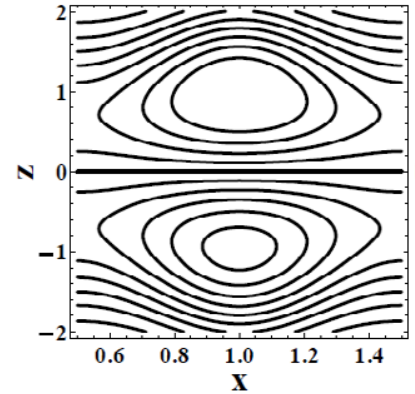

(c)

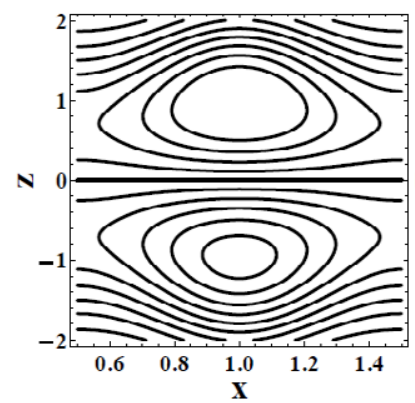

(c)

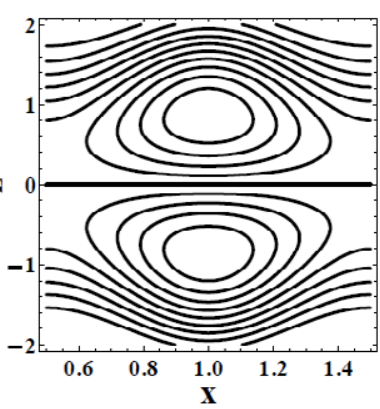

$(d)$

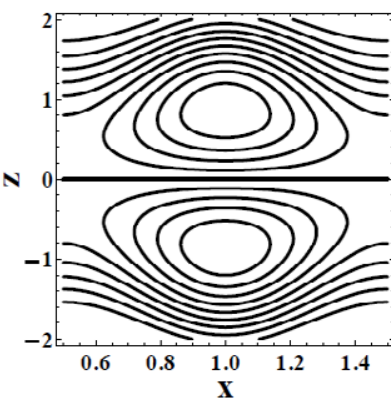

$(d)$
Figure 15: Stream lines for different values of $\alpha$ (a) for $\alpha=0.1$, (b) for $\alpha=0.15$ (c) for $\alpha=0.2$, (d) for $\alpha=0.27$. The other parameters are $y=0.3, \beta=0.5, \beta_{1}=0.5$, $\mathrm{Q}=1, \phi=0.1$
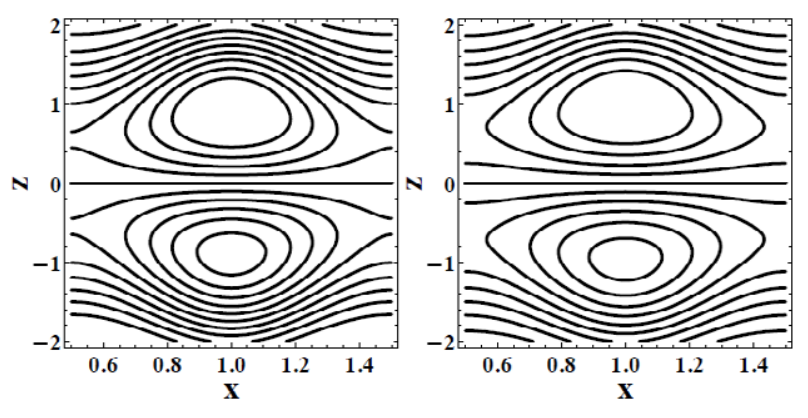

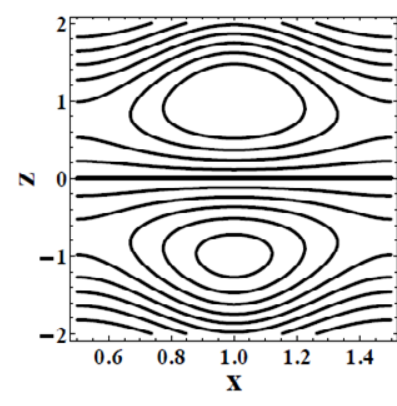

(c)

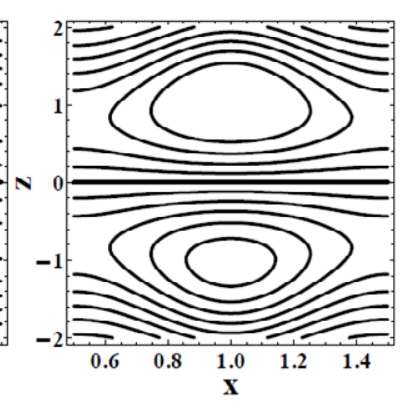

(d)
Figure 16: Stream lines for different values of $\beta_{1}$ (a) for $\beta_{1}=0.4$, (b) for $\beta_{1}=0.5$ (c) for $\beta_{1}=0.6$, (d) for $\beta_{1}=0.8$. The other parameters are $y=0.3, \alpha=0.2, \beta=0.5$, $\mathrm{Q}=1, \phi=0.1$.

6 along the average volume flow rate $\mathrm{Q}$. It is mentioning that pressure rise is increasing in the retrograde pumping region $(\Delta \mathrm{p}>0, \mathrm{Q}<0)$ with

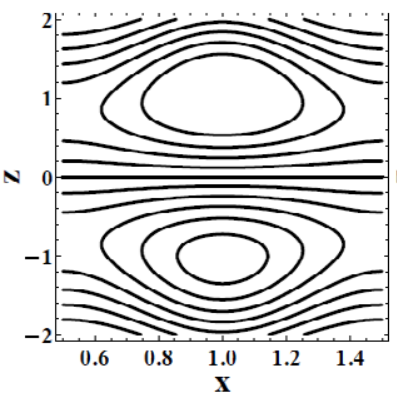

(a)

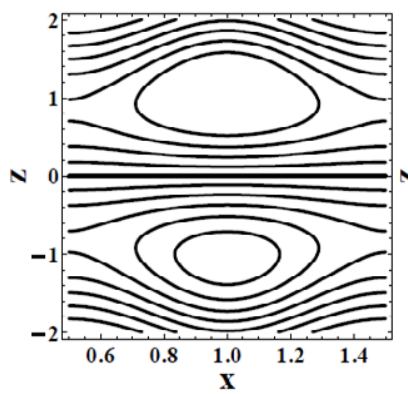

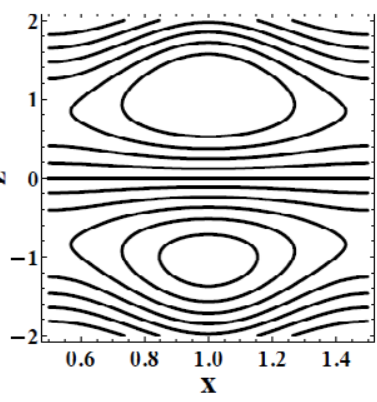

(b)

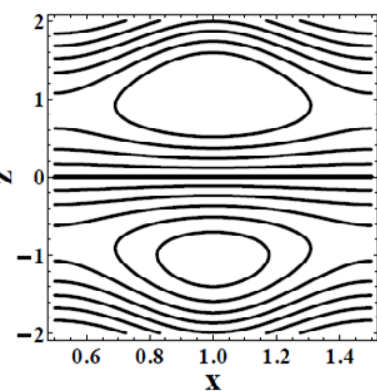

Figure 17: Stream lines for different values of $Q$ (a) for $Q=0.5$, (b) for $Q=1$, (c) for $Q=1.5$, (d) for $Q=2$. The other parameters are $y=0.3, \alpha=0.1, \beta=0.5, \beta=0.5$, $\phi=0.1$.

the increase of aspect ratio and decreases in the peristaltic pumping region ( $\Delta \mathrm{p}>0, \mathrm{Q}>0$ ) and augmented pumping region $(\Delta \mathrm{p}<0, \mathrm{Q}<0)$. It is depicted from Figure 7 that pressure rise curves are getting altitude for the Prandtl fluid constant $\alpha$ in peristaltic pumping and retrograde pumping region while inverse behavior is achieved for pressure rise variation in the co-pumping area of the flow rate. Figure 8 reveals the effect of parameter $\beta$ 1on pressure rise distribution $\Delta p$ across the domain of flow rate $\mathrm{Q}$. It is depicted here that pressure rise is diminishing with the increasing sequence of $\beta_{1}$ for all the values of flow rate $Q$. It is also obtained here that no peristaltic pumping is achieved with the variation of $\beta_{1}$. The behavior of pressure rise is very similar for the amplitude ratio as that of the aspect ratio $\beta$ but the height of the curves does not increase with so much extent as seen for $\beta$ (Figure 9).

Figure 10 contains the variation of pressure gradient profile $\mathrm{dp} /$ $\mathrm{dx}$ for the aspect ratio parameter $\beta$ drawn along the coordinate $\mathrm{x}$. It is noted here that pressure gradient distribution is reduced with the increase in aspect ratio in the region $0.4<\mathrm{x}<0.6$ while increasing in the remaining parts. It is also measured from this figure that much pressure gradient is needed to conserve the flow in the central part of the flow as compared with the boundaries of the rest of the part. It is disclosed in Figure 11 that pressure gradient is linearly varying with the large values of the parameter $\alpha$ in the part $0.4<\mathrm{x}<0.6$ while a very small variation in pressure is observed in the remaining area of the domain $0<x<1$. Figure 12 comprises the graphical features of $\beta_{1}$ on the pressure gradient distribution $\mathrm{dp} / \mathrm{dx}$ and implies that pressure gradient is inversely proportional to the magnitude of $\beta_{1}$ throughout the domain but variation in pressure is almost negligible at the edges of the boundary. We can describe from Figure 13 that pressure gradient is decreasing with the flow rate in the middle of the domain and becomes stable at the corners. It is directed here that more pressure is required to maintain the average flow rate. 
Citation: Nadeem S, Riaz A, Ellahi R (2014) Series Solution of Three Dimensional Peristaltic Flow of Prandtl Fluid in a Rectangular Channel. J Appl Mech Eng 3: 139. doi:10.4172/2168-9873.1000139

Page 7 of 7

Trapping bolus process is investigated in Figure 14 for the aspect ratio $\beta$. It is analyzed here that number of bolus is decreasing with the increase in magnitude of aspect ratio as we see that volume of the bolus is gradually minimized and for $\beta=0.8$, we have only one bolus left. If one examines the Figure 15, he will come to know that size of the bolus is approaching its minimum magnitude but number of bolus is in linear relation with the Prandtl fluid parameter $\alpha$. One can easily observe that for $\alpha=0.1$, we have two boluses but for $\alpha=0.27$, we get four boluses but more close to each other as compared with the first one. It can also be observed that $\mathrm{z}=0$ is the point of distinction for upper and lower bolus. The streamlines for the parameter $\beta_{1}$ are sketched in Figure 16. It can be visualized that boluses are reduced in quantity but enlarge their dimensions as we give more magnitude to $\beta_{1}$. It can be extracted from Figure 17 that with the increasing effect of flow rate $\mathrm{Q}$, the streamline boluses are expanding and that is why they start collapsing and get reduced in number.

\section{References}

1. Malik MY, Hussain A, Nadeem S (2009) Flow of a Jeffrey-six constant fluid between coaxial cylinders with heat transfer analysis. Z. Naturforsch A, 56: 345

2. Astarita G,Marrucci G (1974) Principles of non-Newtonian Fluid Mechanics, McGraw Hill, London.

3. Hameed M, Ellahi R (2011) Thin Film flow of non-Newtonian MHD fluid on a vertically moving belt. Int. J Number Meth Fluids 66: 1409-1419.

4. Shukla PK, Rahman HU (1998) The Rayleigh-Taylor mode with sheared plasma flows. Phys Scripta 57: 286.

5. Mahomed FM, Hayat T (2007) Note on an exact solution for the pipe flow of a third grade fluid. Acta Mech 190: 233-236.
6. AbdElnaby MA, Haroun MH (2008) A new model for study the effect of wall properties on peristaltic transport of a viscous fluid. Commun. Nonlinear Sci. Number. Simul 13: 752-762.

7. Mekheimer KS (2008) Effect of the induced magnetic field on peristaltic flow of a couple stress fluid. Phys Lett A 372: 4271-4278.

8. Tripathi D (2011) A mathematical model for the peristaltic flow of chyme movement in small intestine. Math Biosci 233: 90-97.

9. Srivastava LM, Srivastava VP (1985) Peristaltic transport of a non-Newtonian fluid: application to the vas deferens at small intestine. Ann Biomed Eng 13: 137-53.

10. Ebaid A (2008) Effects of magnetic field and wall slip conditions on the peristaltic transport of a Newtonian fluid in an asymmetric channel. Phys Lett A 372: 4493-4499.

11. Subba Reddy MV, Mishra M, Sreenadh S, Rao AR (2005) Influence of lateral walls on peristaltic flow in a rectangular duct. J Fluids Eng 127: 824-827.

12. Mekheimer KS, Abdelmaboud Y, Abdellateef Al (2012) Peristaltic transport through an eccentric cylinders: Mathematical. modeling, Appl Bionics Biomech 10: $19-27$.

13. Vivian A, Ricardo C, Lisa $F$ (2011) Stokesian peristaltic pumping in a threedimensional tube with a phase-shifted asymmetry. Phys. Fluids, 23: 081901-10.

14. Tang D (1994) Three dimensional numerical and asymptotic solutions for the peristaltic transport of a heat-conducting fluid. Acta Mech 104: 215-230.

15. Patel M, Timol MG (2010)The stress-strain relationshipfor visco-inelastic nonNewtonian fluids. Int. J Appl Math Mech 6: 79-93.

16. He JH (2010) A note on the homotopy perturbation method. Therm. Sci., 14 565-568.

17. He JH (2006) Homotopy perturbation method for solving boundary value problems. Phys Lett A 350: 87-88. 